\title{
A 2.4 GHz Switched Beam Antenna for Indoor Applications Using Angle of Arrival Estimation
}

\author{
Reham Elsamnty¹, Ahmed Mohamed Attiya², Esmat Abdel Fattah Abdallah³, Abdel Hadi Ammar ${ }^{1}$ \\ ${ }^{1}$ Electrical Engineering Department, Al-Azhar University, Cairo, Egypt \\ ${ }^{2}$ Microwave Engineering Department, Electronics Research Institute, Giza, Egypt \\ ${ }^{3}$ Microstrip Department, Electronics Research Institute, Giza, Egypt \\ Email: rehamelsamnty@yahoo.com
}

How to cite this paper: Elsamnty, R., Attiya, A.M., Abdallah, E.A.F. and Ammar, A.H. (2016) A $2.4 \mathrm{GHz}$ Switched Beam Antenna for Indoor Applications Using Angle of Arrival Estimation. Open Journal of Antennas and Propagation, 4, 109-118. http://dx.doi.org/10.4236/ojapr.2016.43008

Received: June 17, 2016

Accepted: August 12, 2016

Published: August 15, 2016

Copyright $\odot 2016$ by authors and Scientific Research Publishing Inc. This work is licensed under the Creative Commons Attribution International License (CC BY 4.0).

http://creativecommons.org/licenses/by/4.0/ (c) () Open Access

\begin{abstract}
This study introduces a new design of planar antenna array for ISM band applications at $2.4 \mathrm{GHz}$. This prototype is switched beam antenna, namely planar antenna with four meandered slotted triangular elements (PAFMSTE), which is proposed for wireless indoor applications. The Base Station (BS) equipped with this planar antenna is preferred to be at the center position on the room ceiling to cover all sectors of the room. It is designed to use four directional triangular elements arranged to form a square planar antenna array. The PAFMSTE is used to obtain optimal directivity in four directions in azimuth plane with $3 \mathrm{~dB}$ beamwidth of $90^{\circ}$ at a specific orientation of $30^{\circ}$ in elevation plane. Switched parasitic technique is used to enable PAFMSTE to steer a directional beam through four locations by using four PIN diodes switches. The High Frequency Structure Simulator (HFSS) is used as an efficient simulation tool to optimize the performance of the PAFMSTE antenna. The fabrication and measurements of the PAFMSTE antenna are introduced. The proposed antenna enable radio positioning via Angle of Arrival (AOA) information collected from nearby devices. Then, the Cramér-Rao Bound (CRB) is presented for AOA estimation using identically and equally spaced antenna elements. The CRB depends on the directivity, where the maximum values of $\mathrm{CRB}$ are $1.35 \times 10^{-3}$ and $8 \times 10^{-3}$ at HPBW of $60^{\circ}$ and $90^{\circ}$, respectively.
\end{abstract}

\section{Keywords}

Indoor Communications, Switched Beam Antenna, Directional Microstrip Antenna, Slotted Patch Antenna, Radio-Positioning, AOA Estimation, CRB

\section{Introduction}

Smart antenna technology is becoming one of the most effective utilities for improving 
performance and available spectrum usage demanded by the rapid growing market of wireless communications [1]. Recently, pattern reconfigurable antennas have received much attention because of their interesting performance in reconfiguring radiation patterns with a single antenna [2]. Many indoor applications are expected to sense the user's position by radio-positioning systems to implement context-aware services that facilitate access to nearby resources, deliver location sensitive information, and enforce proximity based security policies, where the use of GPS is neither cost effective nor technically feasible [3]. Antenna arrays can be used as access points mounted in the ceiling and facing down to support indoor positioning. Figure 1 shows the typical application of the smart planar antenna array for wireless local area network (WLAN) applications.

A switched-beam system is the simplest smart antenna technique. Such an antenna system detects signal strength, chooses from one of several predetermined fixed beams, and switches from one beam to another as the node moves throughout the sector [4]. It has less intensive signal processing and at the same time the installation costs are lower when compared to adaptive antenna technology [5].

This paper presents a new switched beam antenna designed to address the challenges of indoor positioning and suitable for Angle-of-arrival (AOA) estimation. Among the many solutions proposed, systems based on AOA estimation have several qualities that make them attractive to a wide range of applications. Additionally, angle-based positioning only requires measurements from two anchor nodes [6]. The proposed AOA localization system implements a fine-grained positioning and single-anchor localization in azimuth plane without requiring knowledge of the propagation model for the $\mathrm{RF}$ signal. The AOA algorithm embedded system is first estimated and then different parameters in the system are adjusted in accordance with the desired steering angle [7]. Additionally, the signals received by the various antenna elements need to be angularly decorrelated to enable target localization based on AOA estimation [3].

A Base Station (BS) equipped with a switched-beam directional antenna can be optimized for installation on the ceiling of any large indoor space as shown in Figure 1. The center position of BS is unobtrusive to the users and is preferred to serve several nodes. The antenna is implemented by combining four triangular faces pointing in different directions. By switching between different faces, the BS can establish preferred communication with groups of devices located in the area illuminated by each antenna, which reduces the probability of interferences in dense networks [3].

In this scenario, a new electronically beam steerable microstrip patch antenna is presented, which can be mounted in ceilings for indoor wireless network applications. The proposed antenna is reconfigurable by using four PIN diodes switches. A planar antenna with four meandered slotted triangular elements (PAFMSTE) is proposed to cover the room with radiation pattern diversity and high reliability. The radiation pattern direction of PAFMSTE is in the desired direction of $30^{\circ}$ in elevation plane with $3 \mathrm{~dB}$ beamwidth of $90^{\circ}$ in azimuth plane, which covers almost half of the whole solid steradian. 


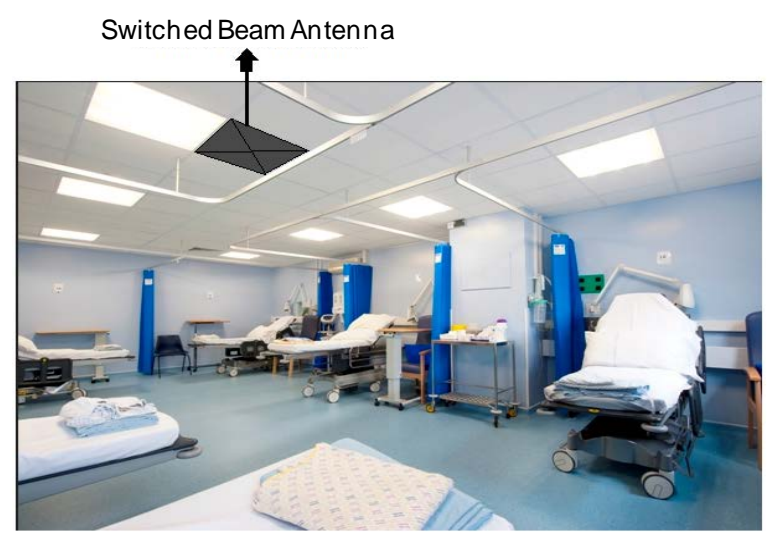

Figure 1. Application for indoor WLAN system by using smart patch antenna.

Design and performing the simulation analysis of the proposed PAFMSTE with the FR-4 epoxy PCB substrate of dimensions $69 \times 69 \times 1.6 \mathrm{~mm}^{3}$ is presented. This antenna is designed to operate at $2.4 \mathrm{GHz}$ ISM band and a $50 \Omega$ microstrip feed line is used. The presented smart antenna is based on 2D planar antenna with high coverage area.

The design of the PAFMSTE antenna is optimized using the finite element method (FEM) utilized in Ansoft's High Frequency Structure Simulator HFSS 10.1.2 [8]. The fabrication and measurements of the PAFMSTE antenna are introduced. The measurements have been performed with a vector network analyzer HP8719ES.

This antenna support directional communication to enable spacial reusability to mitigate multipath propagation. The system can locate a target by estimating AOA of the incoming messages in the azimuth plane, which relies on the received signal strength indicator (RSSI) of the attached base station. Then, the Cramér-Rao Bound (CRB) is presented for AOA estimation using identically and equally spaced antenna elements, where the design parameters are the number of antenna elements and their directivity $[6]$.

\section{Antenna Array Design and Configurations}

The square patch antenna is divided into four identical isosceles triangular antennas and each triangular antenna covers each sector of the room. The single beams should exhibits a directional pattern to reduce the interferences and enable spatial reusability. To meet the design requirements, the PAFMSTE has been identified as a suitable geometry for the antenna implementation as shown in Figure 2. The PAFMSTE is a planar antenna with a simple design and minimum number of elements, which covers all the room as compared to the proposed configuration in [3] [9].

The PAFMSTE consists of four equal size meandered slotted triangular patch elements situated on top of a square printed circuit board (PCB) and with a ground plane located on the bottom side of the PCB forming the well known parallel topology. Thus, the beam-steering can be carried out equally through four directions in azimuth plane, where the beam orientation and coverage can be controlled by four PIN diodes varying 


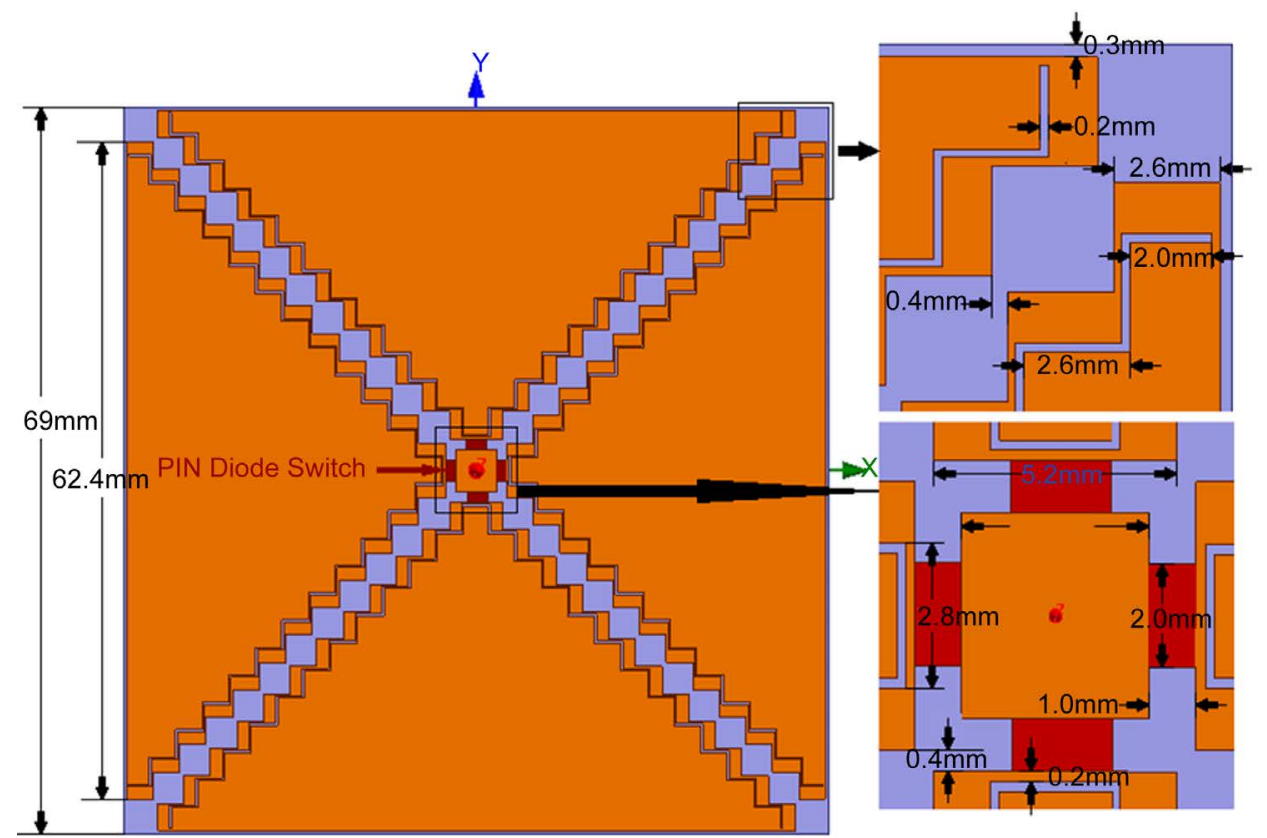

Figure 2. The configuration of the smart patch antenna PAFMSTE.

switches. Each direction state has one PIN diode ON and the others are OFF.

Each isosceles triangular element of the patch antenna has two meandered edges with meandered slot. To obtain the optimal configuration, the patch antenna was optimized with the steepest gradient optimization procedure. By extending the meandered edges of the staircase triangular patch antenna, it becomes equilateral triangular microstrip patch antenna with length of $62.4 \mathrm{~mm}$. The resonance frequency of the equilateral triangular microstrip patch antenna depends on substrate thickness, relative dielectric constant, side length of the equilateral triangle, and the location of the feed point [10]. Loading the slot in the radiating triangular patch antenna can cause meandering of the excited patch surface current paths and result in lowering the antenna fundamental resonant frequency, which corresponds to reducing antenna size as compared to the conventional triangular patch antenna at the same operating frequency [11]. A meandered structure of PAFMSTE is one solution to overcome the size restriction and also the path length of the surface current increases due to an increase in the dimensions of the edges and slots of the four triangular elements.

RF PIN diodes can be used as switches of the reconfigurable radiation pattern antennas. At RF frequencies the MPP4204 PIN diode behaves as a variable resistor, but with slightly more complicated equivalent circuit models for the ON/OFF states, as shown in Figure 3. Both the ON and OFF states have a package inductance $\mathrm{L}=0.2 \mathrm{nH}$. The equivalent circuit for the ON state (forward biased) has a low resistance $\mathrm{R}_{S}=2 \Omega$ which contributes to the insertion loss. The equivalent circuit for the OFF state (zero or reverse biased) has the parallel combination of the parallel reverse bias resistance $R_{P}=5$ $\mathrm{M} \Omega$ and the total capacitance $\mathrm{C}_{\mathrm{T}}=0.15 \mathrm{pF}$, which contributes to the isolation [12]. The PIN diode is modelled in HFSS using two series lumped RLC boundary conditions. The 


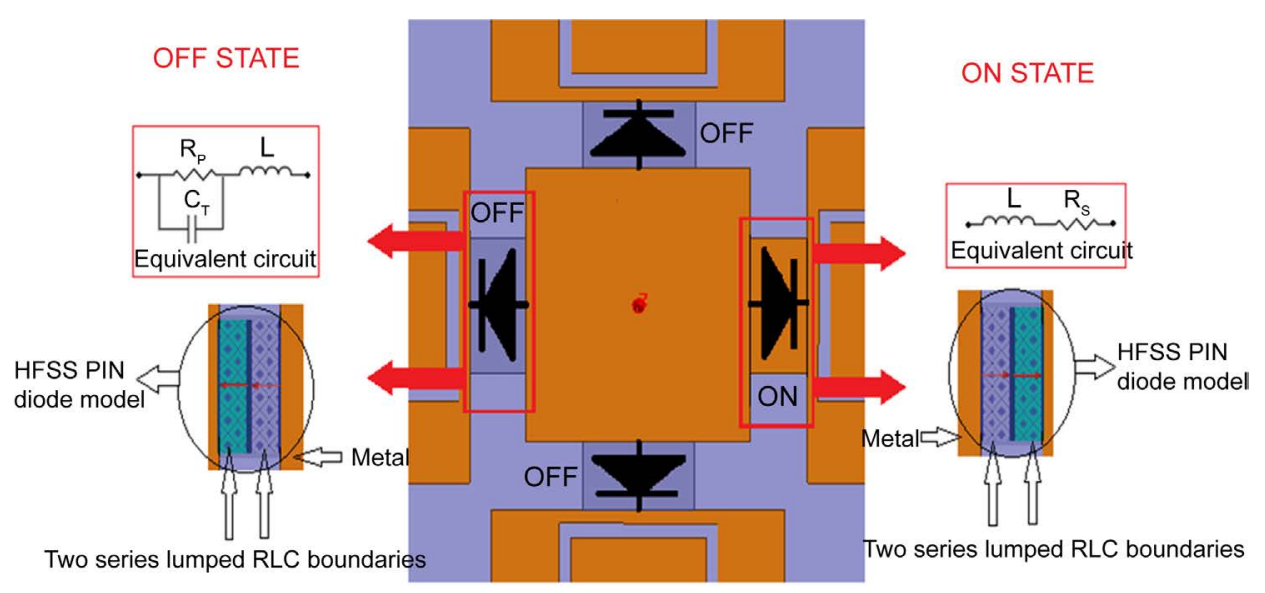

Figure 3. Four RF PIN diodes with their equivalent circuits and HFSS models for the ON/OFF states.

first one is $\mathrm{L}$ and the second one is either the $\mathrm{R}_{S}$ for the $\mathrm{ON}$ state or the parallel combination of $\mathrm{R}_{\mathrm{P}}$ and $\mathrm{C}_{\mathrm{T}}$ for the OFF state, as shown in Figure 3.

\section{Simulated and Measurement Results}

The designed antenna was fabricated on the FR-4 epoxy PCB substrate with a thickness of $1.6 \mathrm{~mm}$, a dielectric constant of 4.4, and a dielectric loss tangent of 0.02 [1]. The fabricated PAFMSTE antenna and its measurement are shown in Figure 4.

Simulation and measurements were performed for the patch antenna with one element fed by a $50 \Omega$ coaxial feed, while the remaining elements open-circuited. The simulated and measured reflection coefficient as a function of frequency was obtained as shown in Figure 5. The good agreement between the measurement and the simulation results are clearly observed, where the simulated and measured return loss of $-27 \mathrm{~dB}$ and $-29 \mathrm{~dB}$, respectively at $2.4 \mathrm{GHz}$ and a $-10 \mathrm{~dB}$ return loss bandwidth of $50 \mathrm{MHz}$ are achieved for PAFMSTE antenna.

Each isosceles triangular patch antenna exhibits a mono-lobe radiation pattern characterized by half power beamwidth in elevation plane of about $60^{\circ}$ and half power beamwidth in azimuth plane of about $90^{\circ}$, which meets the requirements of having a cumulative radiation with an (approximately) uniform coverage.

Figure 6 shows the simulated 3D radiation pattern of each element achieved by shorting one PIN diode switch to connect an element and leaving the remaining PIN diodes switches OFF to disconnect the other elements. The main beam lobe is steered in different directions in azimuth plane.

The simulated far field radiation patterns in the elevation plane and the azimuth plane are illustrated in Figure 7(a) and Figure 7(b), respectively while the main beam lobe is steered in different directions in azimuth plane. The characteristics of the radiation pattern in the elevation and azimuth planes such as elevation angle, maximum directivity, and $3 \mathrm{~dB}$ beam-width are shown in Figure 7. A comparison between simulated and measured radiation pattern in the elevation plane is shown in Figure 8. The 

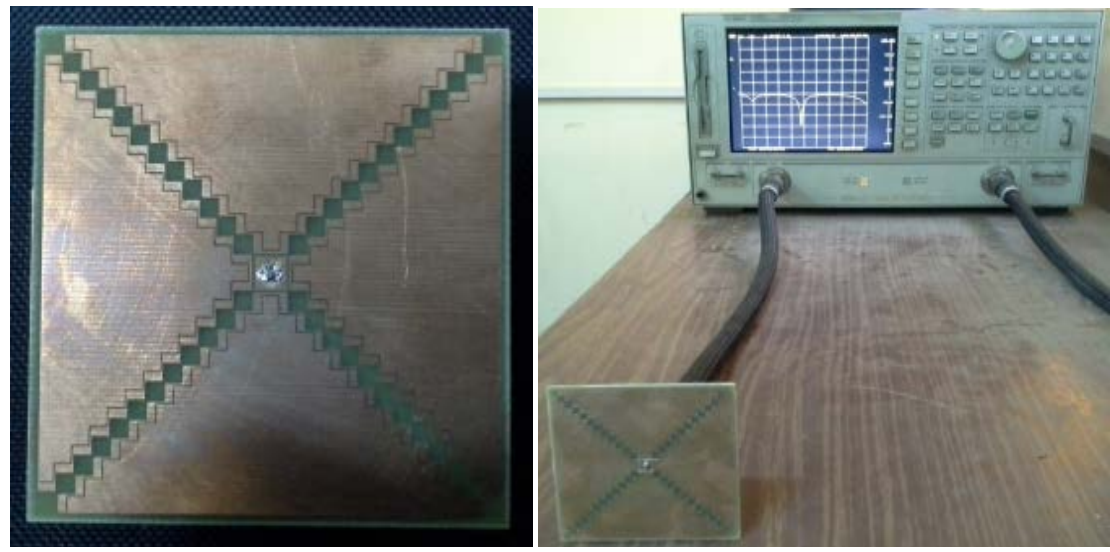

Figure 4. The fabricated PAFMSTE antenna and its measurement.

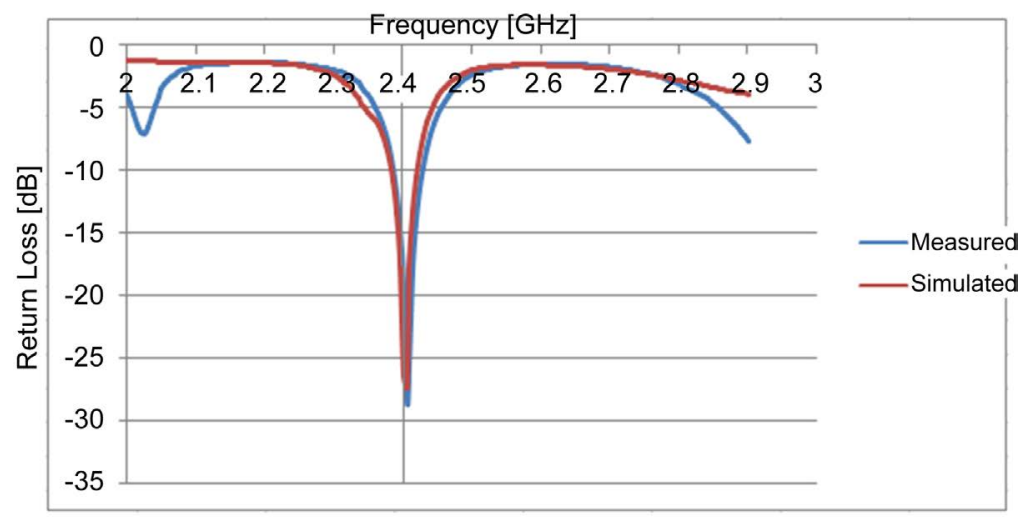

Figure 5. Simulated and measured reflection coefficient of PAFMSTE with one element shortcircuited to the feed.

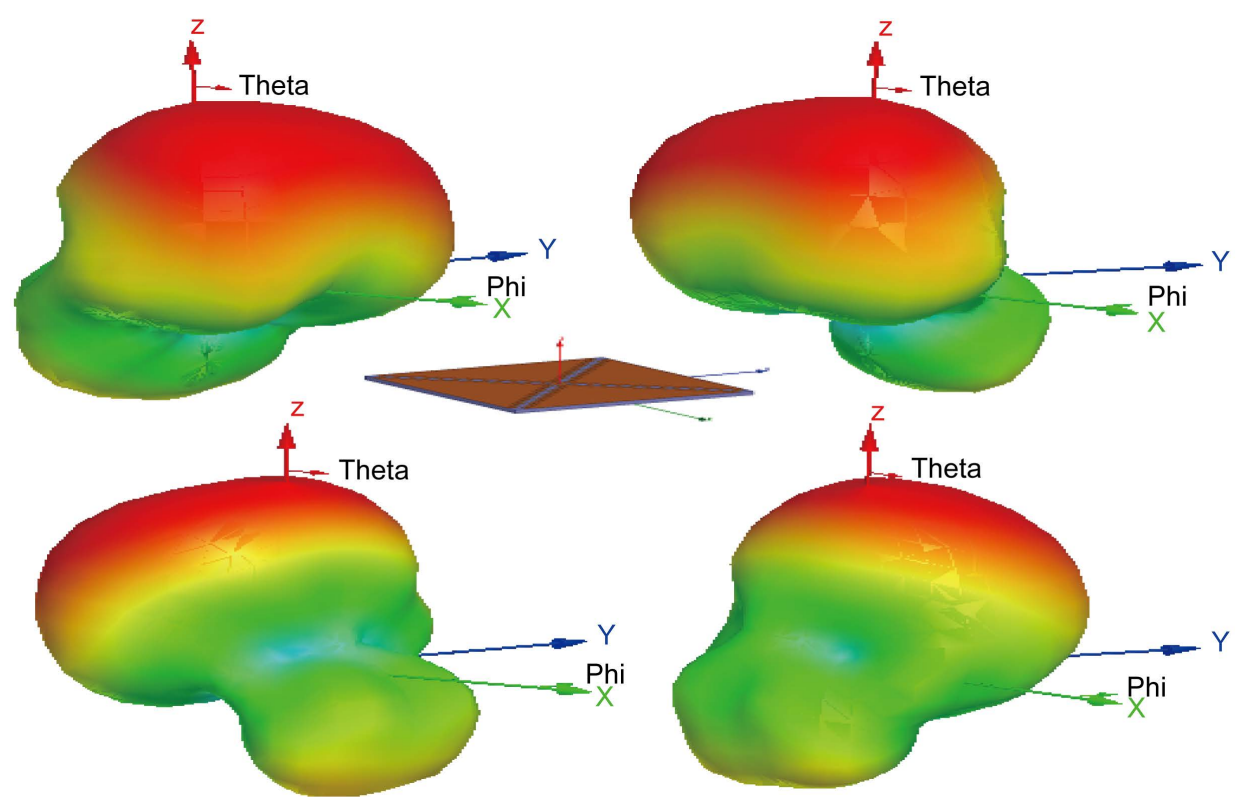

Figure 6. Simulated 3D radiation pattern of PAFMSTE from each antenna element for different switching states. 


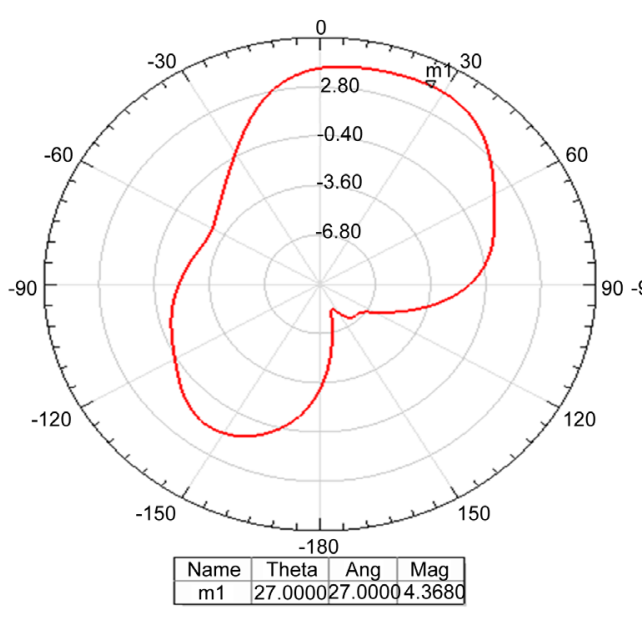

(a)

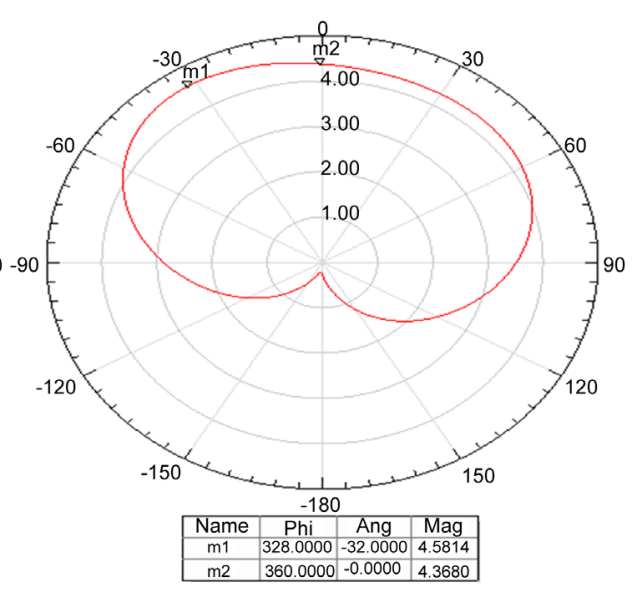

(b)

Figure 7. Radiation patterns of in: (a) elevation plane; (b) azimuth plane.

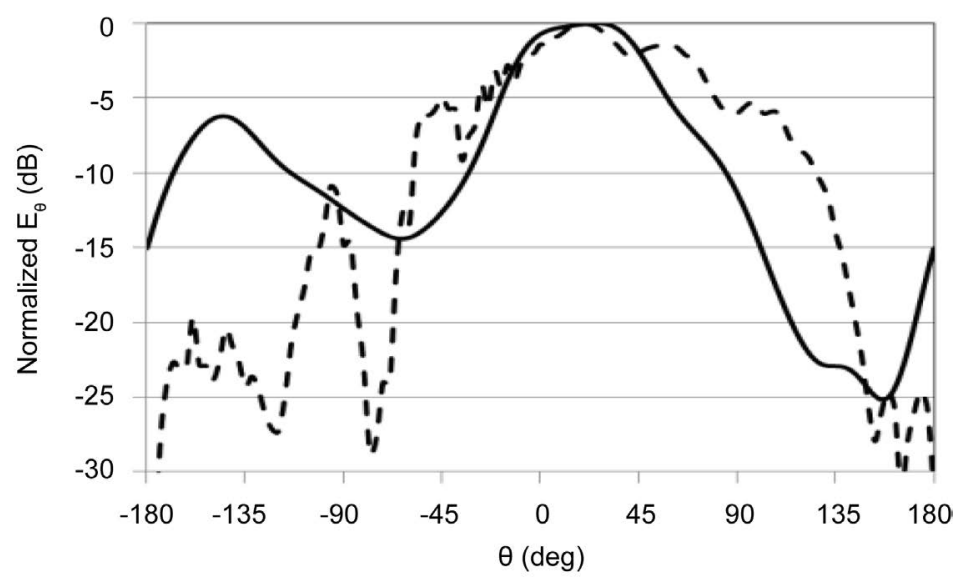

Figure 8. Comparison between simulated (solid line) and measured (dashed line) in the elevation plane.

good agreement between the measurement and the simulation results in the region of interest from $0^{\circ}$ to $90^{\circ}$ are clearly observed. However the holding ground plane in the measuring setup reduced the back radiation compared with the simulated results.

On the other hand, combining the four patches by shorting the four PIN diodes switches to the coaxial feeding point produces nearly omnidirectional pattern as shown in Figure 9.

\section{Angle of Arrival Estimation}

Consider a BS equipped with $N$ selectable antenna beams. The system is designed to estimate the AOA of the messages transmitted by a target node relative to azimuth plane.

To this purpose, the BS measures the Received Signal Strength (RSS) on each triangle element $i$.

The RSS of each element is function of the gain of each element in the direction of the target $G_{i}(\theta)$, the RF power arriving at the BS is $P_{r x}$, and a random component that 


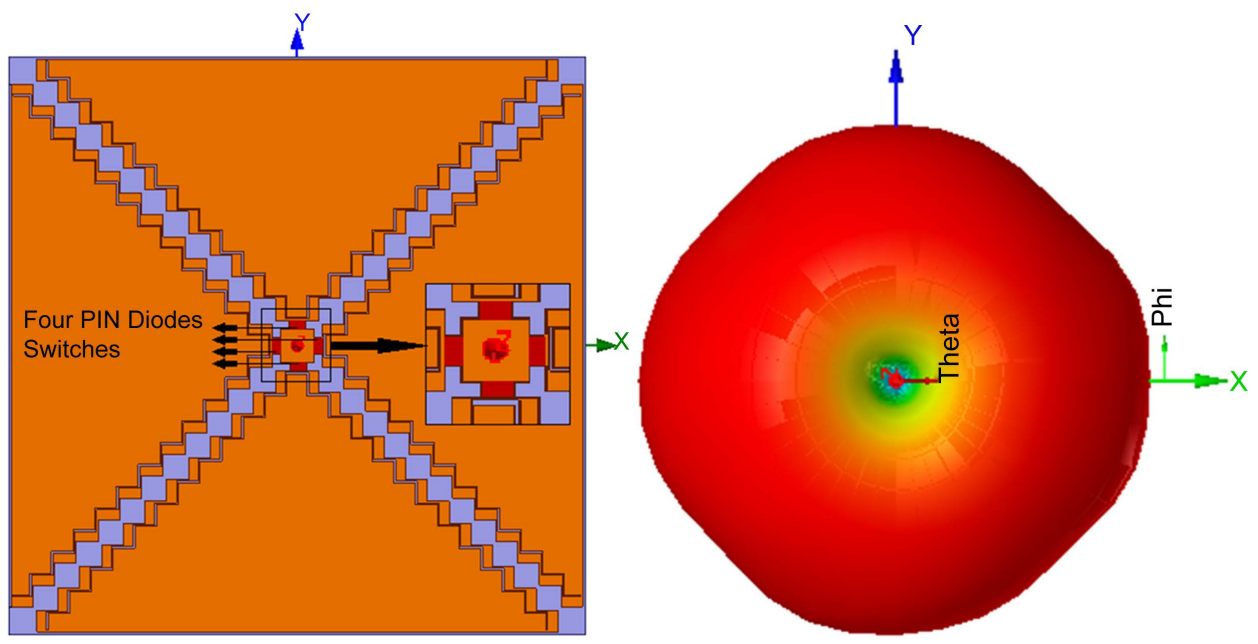

Figure 9. Omnidirectional pattern when all patches are ON.

models the noise is $n_{i}$. Note that each measurement $R S S_{i}$ is a random variable that depends on the unknown parameters $\theta$ and $P_{r x}$.

The $P_{r x}$ value varies with the transmission power used by the target and with the random attenuation introduced by the radio channel. Since $P_{r x}$ does not carry information on $\theta$, its contribution can be removed by considering the $R S S_{i, j}$ differences between two faces $i$ and $j$ and $n_{i j}=n_{i}-n_{j}[6]$.

The PAFMSTE system is built with $N=4$ identical and equally spaced antenna elements, and if the target's distance is much larger than distance between the faces, the gains can be expressed as $G_{i}(\theta)=G(\theta-2 i \pi / N)$.

The analysis adopts the Fisher information $(F)$ to evaluate the accuracy of a generic AOA estimation algorithm in relation to some important design parameters. The value $F$ measures the amount of information that a random variable carries about an unknown parameter. In the case discussed, the random variables are the $R S S_{i, j}$ values, while the parameter to estimate is $\theta$. The Fisher information is:

$$
F_{i j}(\theta)=E\left\{\left[\frac{\partial}{\partial \theta} \log f_{i j}\left(R S S_{i j} ; \theta\right)\right]^{2}\right\}
$$

where $f_{i j}\left(\operatorname{RSS}_{i j} ; \theta\right)$ is the probability density function that describes the measurements $R S S_{i j}$. Assuming independent and identically distributed noise components $n_{i j}$ is the zero-mean additive Gaussian noise. The Fisher information $F(\theta)$ expresses the theoretical limit achievable when estimating $\theta$. If $\theta^{\prime}$ is an unbiased estimator for $\theta$, the inverse of $F(\theta)$ bounds the minimum variance of the estimation error:

$$
\operatorname{Var}\left\{\theta^{\prime}\right\} \geq F^{-1}(\theta)
$$

The inequality Equation (2), known as the Cramer-Rao bound (CRB), is a limit that applies to any estimator that uses measurements $\operatorname{RSS}_{i j}$ to compute $\theta$. The Fisher information and the CRB depend on the gains $G_{i}$ of the antennas used in the system. The gain of each antenna element [13]: 


$$
\begin{aligned}
& G_{i}(\theta, \phi)=\left[\frac{1}{2}\left(1+\sin (\phi) \sin \left(\phi_{i}\right) \cos \left(\theta-\theta_{i}\right)+\cos (\phi) \cos \left(\phi_{i}\right)\right)\right]^{2 m}, \\
& i=0,1,2,3
\end{aligned}
$$

Radiation pattern is maximum at $\varphi_{i}=\varphi_{\max }=30^{\circ}$ and $\theta_{i}=\theta_{\max }=\mathrm{i} \pi / 2$ in elevation and azimuth plane, respectively. The larger values of the exponent $m$ correspond to more directive antennas.

The CRB for the estimated unbiased $\theta^{\prime}$ can be expressed as [14]:

$$
C R B(\theta)=\frac{N \sigma_{r s s}^{2}}{N \sum_{i}^{N}\left[\frac{\partial G_{i}}{\partial \theta}\right]^{2}-\left[\sum_{i}^{N} \frac{\partial G_{i}}{\partial \theta}\right]^{2}}
$$

where $\sigma_{\text {rss }}$ is the RSS measurement variance. The CRBs at HPBW of $60^{\circ}$ and $90^{\circ}$ are illustrated in Figure 10(a) and Figure 10(b), respectively. The maximum values of CRB are $1.35 \times 10^{-3}$ and $8 \times 10^{-3}$ at $\mathrm{HPBW}$ of $60^{\circ}$ and $90^{\circ}$, respectively. The CRB depends on the directivity coefficient $m$, where increasing the directivity of each antenna increases the estimation accuracy of the positioning system. The choice of the parameter $m$ should be evaluated by taking into account the sensitivity of the transceiver connected to the antenna elements, where there is a trade off between the directivity and the coverage area of the system.

\section{Conclusion}

This paper introduced a new smart antenna, namely PAFMSTE based on the concept of switched beam capable of operating for indoor mobile wireless systems in the $2.4 \mathrm{GHz}$ band. The PAFMSTE achieves optimal directivity in four directions in azimuth plane with $3 \mathrm{~dB}$ beamwidth of $90^{\circ}$ at a specific orientation of $30^{\circ}$ in elevation plane to cover all sectors of the room. It is based on 2D planar antenna instead of 3D arrangement. The design approach has been described in details with the PIN diode switches and their HFSS models. The simulated and measured results are described. The proposed solution also showed the AOA estimation of the target node by using the Fisher infor-

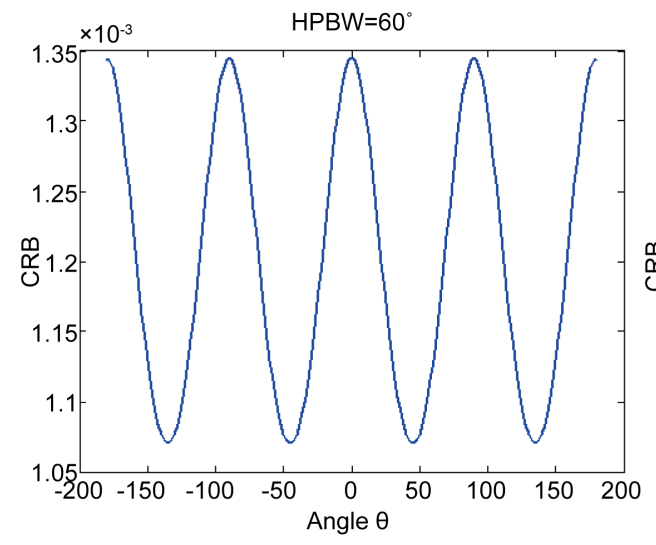

(a)

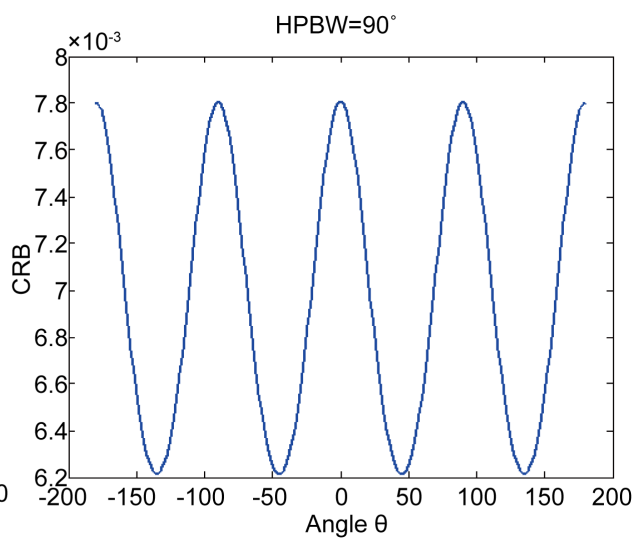

(b)

Figure 10. The CRBs of PAFMSTE with $\sigma_{r s s}=1$ and HPBW of: (a) $60^{\circ}$; (b) $90^{\circ}$. 
mation theory and the CRB. The CRB exhibits good results, where it has maximum values of $1.35 \times 10^{-3}$ and $8 \times 10^{-3}$ at HPBW of $60^{\circ}$ and $90^{\circ}$, respectively. In the future studies, the number of antenna elements will be increased with decreasing the HPBW of each antenna element to improve the CRB values.

\section{References}

[1] Shi, M., Lu, J. and Ireland, D. (2005) Smart Patch Antenna for Indoor Mobile Wireless Computing. Proceedings of IEEE APMC, Suzhou, 3, 4-7 December 2005, 1913-1916.

[2] Tian, H.Y., Li, X.L. and Xia, L.Y. (2013) Beam-Switching Antenna Based on Plane Dipole Structure. Journal of Electronic Science and Technology, 11, 264-267.

[3] Cidronali, A., Maddio, S., Giorgetti, G., Magrini, I., Gupta, S.K.S. and Manes, G. (2009) A $2.45 \mathrm{GHz}$ Smart Antenna for Location-Aware Single-Anchor Indoor Applications. IEEE IEEE MTT-S International Microwave Symposium Digest, Boston, 7-12 June 2009, 15531556. http://dx.doi.org/10.1109/mwsym.2009.5166006

[4] Mubeen, S., Prasad, A.M. and Rani, A.J. (2012) Smart Antennas It's Beam Forming and DOA. International Journal of Scientific and Research Publications, 2, 1-5.

[5] Balanis, C.A. and Ioannides, P.I. (2003) Smart Antenna Systems for Mobile Communications. École polytechnique fédérale de Lausanne, Lausanne.

[6] Giorgetti, G., Maddio, S., Cidronali, A., Gupta, S.K.S. and Manes, G. (2009) Switched Beam Antenna Design Principles for Angle of Arrival Estimation. IEEE EuWIT Conference, Rome, 28-29 September 2009, 5-8.

[7] Stevanović, I., Skrivervik, A. and Mosig, J.R. (2007) Introduction to Smart Antennas. 1st Edition, San Rafael, Morgan \& Claypool.

[8] High-Frequency Structure Simulator, HFSS v. 10.1.2, Trademark of Ansoft Corporation. Four Station Square, Pittsburgh.

[9] Saleem M. K., Alkanhal, M. A. S. and Sheta, A. F. (2016) Switched Beam Dielectric Resonator Antenna Array with Six Reconfigurable Radiation Patterns. International Journal of RF and Microwave Computer-Aided Engineering, 26, 519-530.

[10] Vishwakarma, R.K., Ansari, J.A. and Meshram, M.K. (2006) Equilateral Triangular Microstrip Antenna for Circular Polarization Dual-Band Operation. Indian Journal of Radio \& Space Physics, 35, 293-296.

[11] Bhomia, Y., Kajla, A. and Yadav, D. (2010) V-slotted Triangular Microstrip Patch Antenna. International Journal of Electronics Engineering, 2, 21-23.

[12] Rayno, J.T. and Sharma, S.K. (2012) Frequency Reconfigurable Spirograph Planar Monopole Antenna (SPMA). Proceedings of IEEE ISAP, Nagoys, 29 October-2 November 2012, 1305-1308.

[13] Cidronali, A., Maddio, S., Giorgetti, G. and Manes, G. (2012) Analysis and Performance of a Smart Antenna for 2.45-GHz Single-Anchor Indoor Positioning. IEEE Transactions on Microwave Theory Techniques, 58, 21-31. http://dx.doi.org/10.1109/TMTT.2009.2035947

[14] Zekavat, R. and Buehrer, R.M. (2012) Handbook of Position Location: Theory, Practice and Advances. 1st Edition, John Wiley and Sons, New York. 
Submit or recommend next manuscript to SCIRP and we will provide best service for you:

Accepting pre-submission inquiries through Email, Facebook, LinkedIn, Twitter, etc. A wide selection of journals (inclusive of 9 subjects, more than 200 journals)

Providing 24-hour high-quality service

User-friendly online submission system

Fair and swift peer-review system

Efficient typesetting and proofreading procedure

Display of the result of downloads and visits, as well as the number of cited articles

Maximum dissemination of your research work

Submit your manuscript at: http://papersubmission.scirp.org/ 